\title{
El historial académico de secundaria como factor predictor del rendimiento universitario. Caso de estudio
}

The High School Academic Record as University Performance Predictive Factor

História acadêmica do ensino médio como preditor de desempenho universitário

Omar Alberto Tapasco-Alzate* iD orcid.org/0000-0002-9751-6737 Francisco Javier Ruiz-Ortega** (iD orcid.org/0000-0003-1592-5535 David Osorio-García*** iD orcid.org/0000-0002-9944-364X

Diógenes Ramírez-Ramírez **** (iD) orcid.org/0000-0002-4533-3883

Para citar este artículo: Tapasco-Alzate, O.A., Ruiz-Ortega, F. J., Osorio-García, D. y Ramírez-Ramírez, D. (2020). El historial académico de secundaria como factor predictor del rendimiento universitario. Caso de estudio. Revista Colombiana de Educación, /(81), 147-170. https://doi.org/10.17227/rce.num81-7530

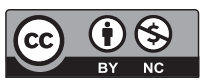

Recibido: 12/02/2018

Evaluado: 01/02/2019

* Magíster en Enseñanza de la Matemática. Profesor en la Universidad de Caldas, Manizales, Colombia. Correo electrónico: omar.tapasco@ucaldas.edu.co

** Doctor en Didáctica de las Ciencias y las Matemáticas. Docente de la Universidad de Caldas, Manizales Colombia; Investigador en el Programa de Reconstrucción del tejido social en zonas de postconflicto en Colombia en Universidad de Caldas, Manizales, Colombia. Correo electrónico: francisco.ruiz@ucaldas. edu.co

*** Magíster en Ciencias Sociales. Docente en la Universidad de Caldas, Manizales, Colombia. Correo electrónico: david.osorio@ucaldas.edu.co

**** Magíster en Estadística. Docente en la Universidad Nacional de Colombia, Bogotá, Colombia. Correo electrónico: ddramirezra@unal.edu.co 


\section{Resumen}

Este artículo de investigación indaga sobre el poder predictivo que el desempeño académico de secundaria tiene sobre el éxito académico en la universidad, al igual que algunas de las implicaciones de su incorporación en el proceso de admisión universitario. La población de esta investigación fueron estudiantes que ingresaron a la Universidad de Caldas en los años 2011-2012. Se acudió a los modelos de regresión múltiple y al cálculo de la importancia relativa de las variables regresoras para medir su capacidac predictiva. Se realizó un análisis comparativo de la incidencia que los resultados en las Pruebas Saber del Icfes tienen en la reproducción de las desigualdades sociales. El modelo obtenido contempla al desempeño en secundaria y al puntaje de admisión como variables predictoras del éxito universitario, explicando un 18,3\% de la variabilidad tota de los datos. La importancia relativa arroja que el 78,3 \% se le atribuye al desempeño en secundaria y el $21,7 \%$ al puntaje de admisión. Los resultados obtenidos son consistentes con múltiples investigaciones en el área, por lo que la recomendación apunta a incorporar el rendimiento académico de secundaria en los procesos de admisión de las instituciones de educación superior.

\section{Palabras clave}

admisión académica; rendimiento académico; previsión; estadísticas educacionales; análisis de regresión

\section{Keywords}

academic admission; academic performance; prediction; educational statistics; regression analysis

\begin{abstract}
This research article investigates the predictive power that high school academic performance has on academic success in university, as well as some of the implications of its incorporation in the university admission process. The population for this research were students who entered the University of Caldas in the years 2011-2012. Multiple regression models and the calculation of the relative importance of the return variables were used to measure their predictive capacity. A comparative analysis of the incidence that the results in the Icfes Pruebas Saber has on the reproduction of social inequalities was carried out. The model obtained considers high school performance and admission score as predictive variables of university success, accounting for $18.3 \%$ of the total variability of the data. The relative importance shows that $78.3 \%$ is attributed to high school performance and $21.7 \%$ to the admission score. The results obtained are consistent with multiple investigations in the area, so the recommendation aims to incorporate high school academic performance in the admission processes of higher education institutions.
\end{abstract}

\section{Palavras-chave}

dmissão acadêmica; desempenho académico; previsão; estatística educaciona; análise de regressão 


\section{Introducción}

Desde hace ya varios años, los sistemas de admisión a la universidad han sido objeto de debate por su baja capacidad para predecir el rendimiento académico de los seleccionados, así como por la pertinencia de los criterios de elección utilizados conducentes a evitar discriminaciones por factores sociales, étnicos, etarios o de género (Ting, 2001; Juarros, 2006). Esta discusión busca dar respuesta a cómo evitar el aumento de las desigualdades sociales entre aquellos estudiantes que, por sus condiciones académicas y socioeconómicas, logran mejores resultados en las pruebas exigidas para su ingreso y aquellos que están predestinados, por los mismos factores, a obtener resultados poco competitivos (Sánchez y Otero, 2012; Rivera y Granobles, 2012).

Se sabe además que los procesos de admisión tienen altas implicaciones para las instituciones, el Estado y la sociedad en general, por lo que cobra especial interés que la universidad evalúe y diagnostique sus procesos y productos, identificando aquellas variables que en mayor medida contribuyan al éxito académico de sus estudiantes, logren su permanencia y, en consecuencia, favorezcan la culminación de los programas académicos por ellos elegidos (Esteban et ál., 2017). En este sentido, uno de los factores que distintos investigadores (Olani, 2009; Cerdeira, Nunes, Reis y Seabra, 2018, entre otros) han reportado tener una incidencia significativa en el éxito académico en la universidad es el desempeño en la educación media, por lo que la presente investigación focaliza su análisis en evidenciar el poder predictivo que el desempeño académico de secundaria tiene sobre el éxito académico en la universidad, al igual que las implicaciones de su incorporación en el proceso de admisión como moderador de la desigualdad social en nuestro contexto.

Para comprender posibles relaciones entre el rendimiento académico de secundaria y el éxito académico de los estudiantes universitarios es necesario conceptualizar y discutir al menos tres aspectos. El primero, el poder predictivo como atributo deseado en un proceso de admisión; el segundo, sobre cómo los sistemas de admisión podrín ejercer como mecanismo de reproducción de la desigualdad social y el tercero, las variables predictivas asociadas a los procesos de admisión que son reconocidas desde la literatura como determinantes del éxito académico.

\section{Poder predictivo}

Entre los aspectos más relevantes a los que le deben apuntar los procesos de admisión está considerar dentro de sus estructuras la valoración aquellas variables que mayor poder predictivo tengan del futuro éxito académico 
en la universidad. Aunque se debe reconocer que la tarea presenta grandes retos dada la complejidad del fenómeno, pues es claro que tener un buen desempeño en la universidad dependerá de múltiples factores de naturaleza individual, familiar, institucional y social, sumándose a los anteriores aquellos de naturaleza macroeconómica, ya que la situación económica del país influirá en la decisión de optar o no por una determinada formación universitaria.

Uno de los criterios a los que se acude con frecuencia en los procesos de admisión es el resultado obtenido por los estudiantes en las pruebas propias que cada institución aplica y en las pruebas estandarizadas realizadas por el Estado, pues trae algunas ventajas prácticas de su tratamiento, como facilidad en su implementación, conocimiento para las universidades del nivel alcanzado por los postulantes en determinadas áreas de conocimiento y su ordenamiento según los desempeños mostrados (Geiser, 2016). Pero tales pruebas no están ausentes de grandes debates y controversias (Sulphey, Al Kahtani, y Abdul Malik Syed, 2018), en las que se exponen problemas sobre el bajo poder predictivo, el significativo impacto negativo en los estudiantes pertenecientes a minorías y el hecho de que estas resulten midiendo la capacidad de los estudiantes de pagar los servicios de preparación para las pruebas (Aguirre y Pabón, 2008; Prakhov, 2015).

Entre las críticas que relacionan el desempeño en las pruebas estandarizadas con el contexto social de los estudiantes, se señala que muchas de ellas son empleadas para proporcionar indicadores de capacidad de razonamiento, resolución de problemas, aprendizaje y adaptación en situaciones complejas que requieren del intelecto, habilidades que son especialmente potencializadas y desarrolladas en las personas de clase social media y alta, y en consecuencia, son los que obtienen mejores puntajes. Mientras que, en la clase social baja y grupos étnicos minoritarios, dichas habilidades son menos cultivadas y registran puntuaciones significativamente más bajas (Bourdieu y Jean-Claude, 2009; Moral de la Rubia, 2006).

Otras de las críticas mencionadas apuntan a que dichas pruebas proporcionan una valoración relativa del desempeño del estudiante con relación a los demás aspirantes que presenten la prueba en una misma cohorte, lo que provoca que tales puntuaciones no resulten adecuadas para la comparación entre cohortes, y consecuentemente, imposibilita la detección de los logros académicos a través del tiempo (Geiser, 2016). Adicionalmente, los estudiantes a menudo son presionados a prepararse para tales pruebas y con relación a ello, muchos expertos académicos argumentan que el énfasis excesivo en las pruebas compromete y distorsiona el sistema educativo en general, pero que, en ausencia de una alternativa viable, tales pruebas de elegibilidad estandarizadas continuarán siendo utilizadas ampliamente en todo el mundo (Sulphey, Al Kahtani, y Abdul Malik Syed, 2018). 
Es de anotar que los procesos de admisión que adjudiquen puntuaciones u ordenaciones con un bajo poder predictivo conducen a errores que pueden clasificarse en dos direcciones. Por un lado, estarán los estudiantes admitidos que resultan beneficiados del proceso de admisión pero que tendrían un desempeño inferior a aquellos que el proceso les niega la oportunidad; por otro, los estudiantes rechazados que podrían haber tenido un desempeño exitoso en la universidad, lo que conduce a problemas de desigualdad y de calidad (Geiser, 2016). En este sentido, Hoare y Johnston (2011) reportan, en el contexto británico, que a pesar de que los estudiantes provenientes de instituciones privadas obtienen mejores resultados en las pruebas estandarizadas que los que asistieron a colegios públicos, no obtenían mejores resultados en los programas universitarios, y que por el contrario presentaban un rendimiento inferior.

\section{Pruebas estandarizadas y reproducción de la desigualdad social}

Es amplia la evidencia recabada que apunta a que los procesos de admisión a la educación superior basados solo en pruebas estandarizadas no están contribuyendo a la reducción de las brechas socioeconómicas de los estudiantes, sino que por el contrario están acentuando tales desigualdades sociales. Más aún, si se tiene en cuenta el rol fundamental que cumple la educación en la movilización social de los países y el gran impacto redistributivo que tiene el nivel educativo alcanzado por los ciudadanos, pues su incremento o una atenuación en su dispersión conducen a la reducción de los indicadores de desigualdad de las naciones (Rivera y Granobles, 2012).

Desde esta perspectiva se identificaron, en el contexto colombiano, los resultados de investigaciones realizadas por Chica, Galvis y Ramírez (2010), y Sánchez y Otero (2012), quienes reportan que los estudiantes rodeados de condiciones socioeconómicas favorables son los que obtienen los mejores puntajes en las Pruebas Saber 11 y, en consecuencia, son los que pueden acceder a una educación superior de mayor calidad, pues las mejores universidades, tanto públicas como privadas, exigen altos puntajes para el ingreso.

En este mismo sentido, el trabajo de Geiser (2016) plantea que, si bien en los estratos bajos hay estudiantes con un rendimiento académico relativamente sobresaliente, dichos puntajes no son tan altos si se les comparan con los de los estudiantes de los estratos 5 y 6, lo que provoca que las puntuaciones en las pruebas estandarizadas actúen como una barrera para impedir el ingreso de estudiantes de contextos desfavorecidos. A este respecto, Sánchez y Otero (2012) reportan que mientras una alta 
proporción de estudiantes de estratos bajos no puede acceder a la educación universitaria o se retira antes de finalizarla, optando en su mayoría por formación técnica o tecnológica, más del 80 \% de los estudiantes de estratos 5 y 6 asiste a una universidad, lo que sugiere que a medida que el retorno económico esperado por educarse aumenta, la participación de los estudiantes de estratos bajos disminuye.

Asimismo, resultados de estudios recientes en otros países (Prakhov y Yudkevich, 2017) arrojan que este tipo de pruebas trae beneficios para las personas con mejor condición socioeconómica, sobre todo si acceden a cursos y pruebas preparatorias.

Para Colombia, tales desigualdades se hacen evidentes al hacerle seguimiento a los resultados comparativos por estratos del promedio de los puntajes de las Pruebas Saber 11 (figura 1), en el que se demuestra una diferencia de 14,8 puntos entre las personas de estrato 6 y las que viven en zona rural; y a su vez, las zonas rurales, los estratos 1,2 y 3 se encuentran con promedios por debajo de 50 puntos, mientras que los estratos 4,5 y 6 se ubican por encima de dicho valor. En pocas palabras, nos encontramos ante una reproducción de desigualdades sociales (Bourdieu y Passeron, 2009) que se disfrazan como "unas desigualdades naturales, desigualdades de talentos" (p. 103); y que mantienen así una lógica que al unísono oculta y admite las diferencias del éxito educativo.

Promedio general pruebas Saber 11

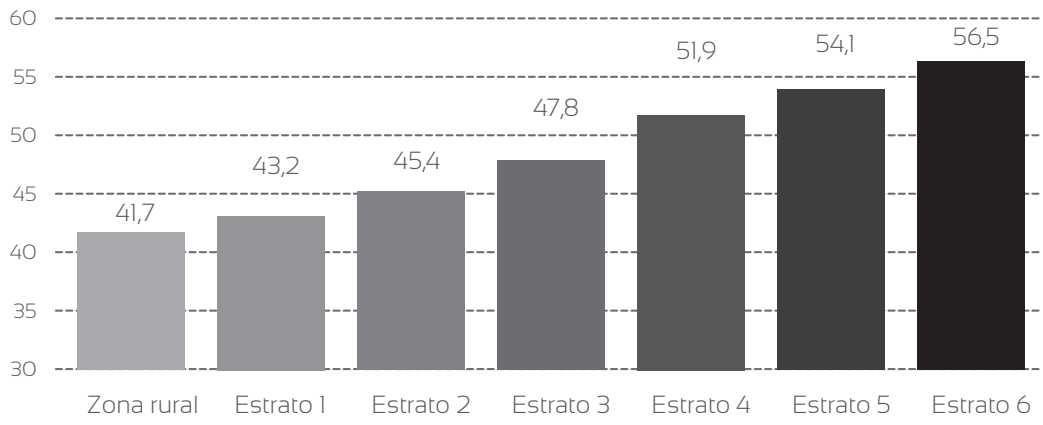

Figura 1. Promedio de los puntajes las Pruebas Saber 11 entre 2008-1 y 2012-2 de toda Colombia

Fuente: elaboración propia a partir de las bases de datos del Icfes.

1 Se excluyeron los valores perdidos de la variable "estrato socioeconómico". Sin estos datos, se tiene 2981327 individuos en la base de datos que presentaron las pruebas en ese periodo de tiempo. Las pruebas con que se calculó el promedio fueron: Biología, Ciencias Sociales, Filosofía, Física, Inglés, Lenguaje, Matemática y Química. 
A su vez, no solo en los estratos socioeconómicos se refleja una estructura de desigualdades sociales a través de las Pruebas Saber 11, también en la relación centro-periferia se mantiene esta situación. En la figura 2 se aprecia el mapa del departamento de Caldas, en el que cada municipio cuenta con un promedio de los puntajes de la prueba, y expone con la intensidad de grises la variación ascendente en los puntajes, es así que, con una tonalidad oscura, se encuentran los municipios cercanos a la capital. Esto quiere decir que las personas residentes en la capital y cercana a ella tienen mejores resultados en las Pruebas Saber 11 para esos años; mientras que la mayoría de municipios que están en la periferia no tienen tan buen desempeño. En este sentido, hay mecanismos de admisión a la educación superior que intentan mitigar este tipo de desigualdad regional, pero Vidigal (2018) expone que las diferencias en el desempeño académico se mantienen, favoreciendo a las regiones que históricamente se localizan cercanas a centros de desarrollo económico.

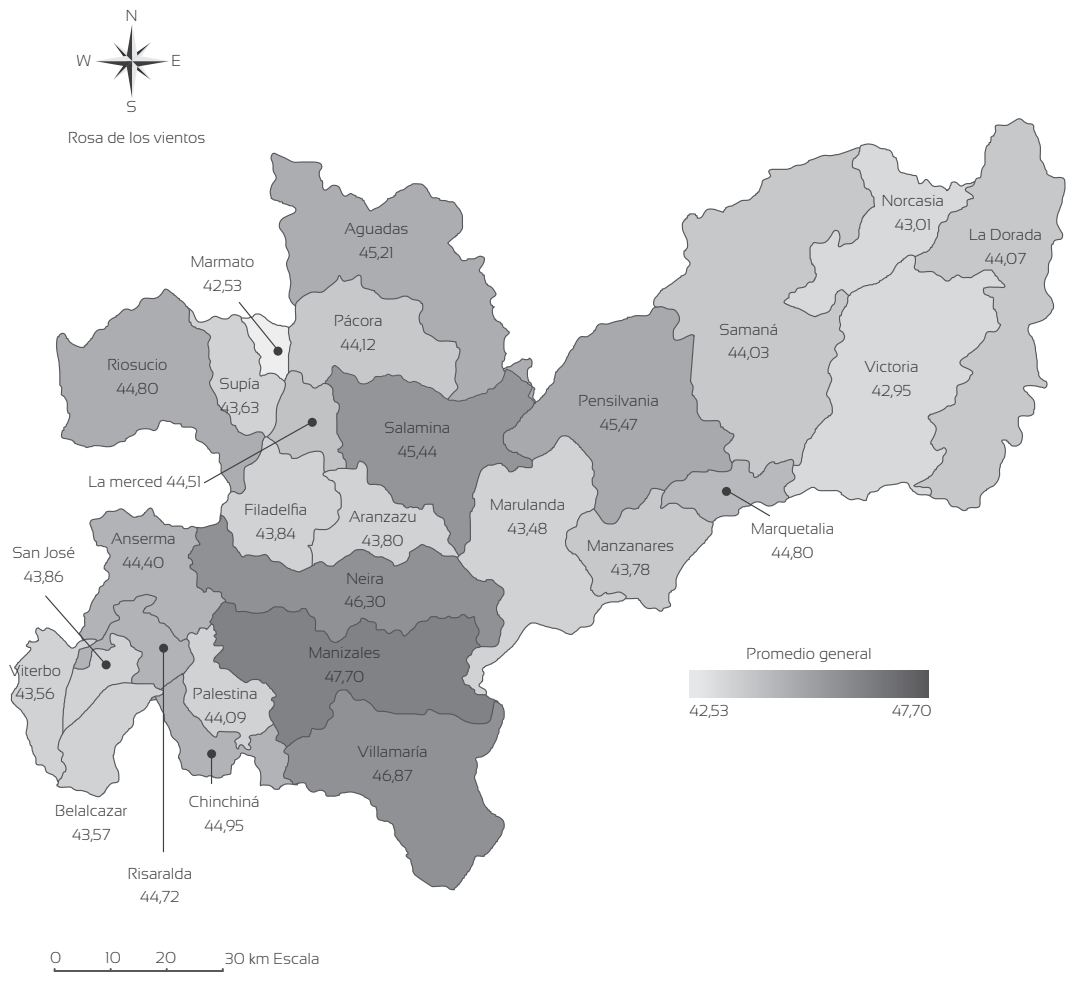

Figura 2. Promedio de los puntajes de las Pruebas Saber 11 entre 2008-1 y 2012-2 por municipio en el departamento de Caldas

Fuente: elaboración propia a partir de las bases de datos del Icfes. 


\section{Variables predictivas}

La literatura referente a estudiar las variables predictivas del desempeño universitario es amplia. Para comenzar, la investigación desarrollada por Ting (2001) hace el llamado a la inclusión de variables psicosociales en las decisiones de admisión, como el autoconcepto, el desarrollo personal, las metas a largo plazo, la participación en actividades extracurriculares y el liderazgo, entre otras. En concordancia, Credé y Kuncel (2008) sugieren que las habilidades, los hábitos, las actitudes y la motivación hacia el estudio son tan determinantes en el desempeño académico como los dos factores predictivos más utilizados: el rendimiento académico previo y las puntuaciones en las pruebas estandarizadas.

Por su parte, Pretz y Kaufman (2015), recomiendan la incorporación de la medición de la creatividad, como variable predictiva del éxito académico no convencional (no cognitivas para ellos) que acompañe los sistemas de admisión. También, en esta misma línea se identificó el trabajo de Pinxten et ál. (2017), quienes manifiestan que, además del rendimiento académico de los estudiantes, sería importante evidenciar la capacidad de regulación que ellos tienen de sus procesos de aprendizaje, como posible indicador de su rendimiento académico universitario, lo que justificaría la revisión de dichos sistemas, en procura de incrementar y enriquecer la selectividad estudiantil a las instituciones de educación superior. Por otro lado, Ishitani y Snider (2006) encontraron que asistir a programas de preparación disminuye la probabilidad de abandonar los estudios universitarios, que los estudiantes provenientes de hogares con mayores ingresos tienen mayor probabilidad de culminar sus estudios y que los alumnos que son primera generación en asistir a la universidad tienen un mayor riesgo de desertar que aquellos estudiantes cuyos padres han pasado por estudios universitarios.

El trabajo de Guzmán (2012), realizado en la ciudad de México, focalizó la atención en una serie de variables, que desde la literatura se han definido como relevantes para la predicción de los desempeños académicos de los estudiantes en los claustros universitarios: género, nivel socioeconómico, edad de la madre, edad del padre, tipo de preparatoria, estudios de la madre y estudios del padre, promedio de la preparatoria, becas obtenidos por los estudiantes o la presentación de pruebas disciplinares. Su estudio ratifica la importante incidencia de las tres últimas, es decir, desempeño en la preparatoria, becas obtenidas y las pruebas disciplinares.

El estudio de Soria y Zúñiga (2014), tuvo en cuenta para su análisis 13 variables explicativas del desempeño académico agrupadas en tres categorías: personales, educación media y resultados de pruebas 
especializadas (matemáticas). Las conclusiones tras el análisis mediante dos modelos logísticos evidenciaron que las variables con mayor poder predictivo, en su orden, fueron: las notas de la educación media, los resultados en las pruebas especializadas y, por último, el desfase entre el año de egreso de las instituciones de educación media y el ingreso a la universidad. En relación con la primera variable, se da especial importancia a que los promedios obtenidos en las instituciones de educación media posiblemente responden a buenas estrategias y métodos de estudio, así como a su disciplina y responsabilidad individual, elementos que en el sistema universitario chileno también son valorados en los procesos académicos.

La investigación realizada por Taylor (1999), orientada hacia la correlación entre los resultados del test diferencial de aptitudes (differential aptitude test, DAT) y el promedio de calificaciones (grade point average, GPA) para estudiantes de primer año de Enfermería, muestra que las calificaciones promedio obtenidas por los estudiantes en su formación secundaria y la prueba de razonamiento verbal tienen una incidencia significativa de su rendimiento en el primer año de estancia universitaria.

El estudio de Ferreyra (2007) muestra que no hay homogeneidad en la incidencia sobre el desempeño académico de los estudiantes de variables como: nivel educativo de sus padres, la condición laboral al ingreso, la situación socioeconómica y la condición laboral de su madre. Situación diferente ocurre con la incidencia del promedio de la formación en la secundaria como factor relevante para comprender y explicar el desempeño de los estudiantes en todos los casos. Por su parte, Moral de la Rubia (2006) reporta que entre 25 posibles variables predictoras del promedio en el semestre, solo cinco mostraron correlación significativa, en las que se encontraron pruebas específicas en capacidad verbal, razonamiento abstracto y numérico, es decir, que miden las capacidades intelectuales de los estudiantes.

A manera de síntesis, en la tabla 1 se sistematizan las investigaciones referenciadas y otras más, con relación a las variables explicativas del éxito académico universitario, referenciadas como celdas de color negro. En la tabla se hace explícita la diferencia entre las variables que son contempladas en los estudios y no resultan ser significativas, señaladas con equis, de las que no son incorporadas en el análisis, correspondiente a las celdas en blanco. 


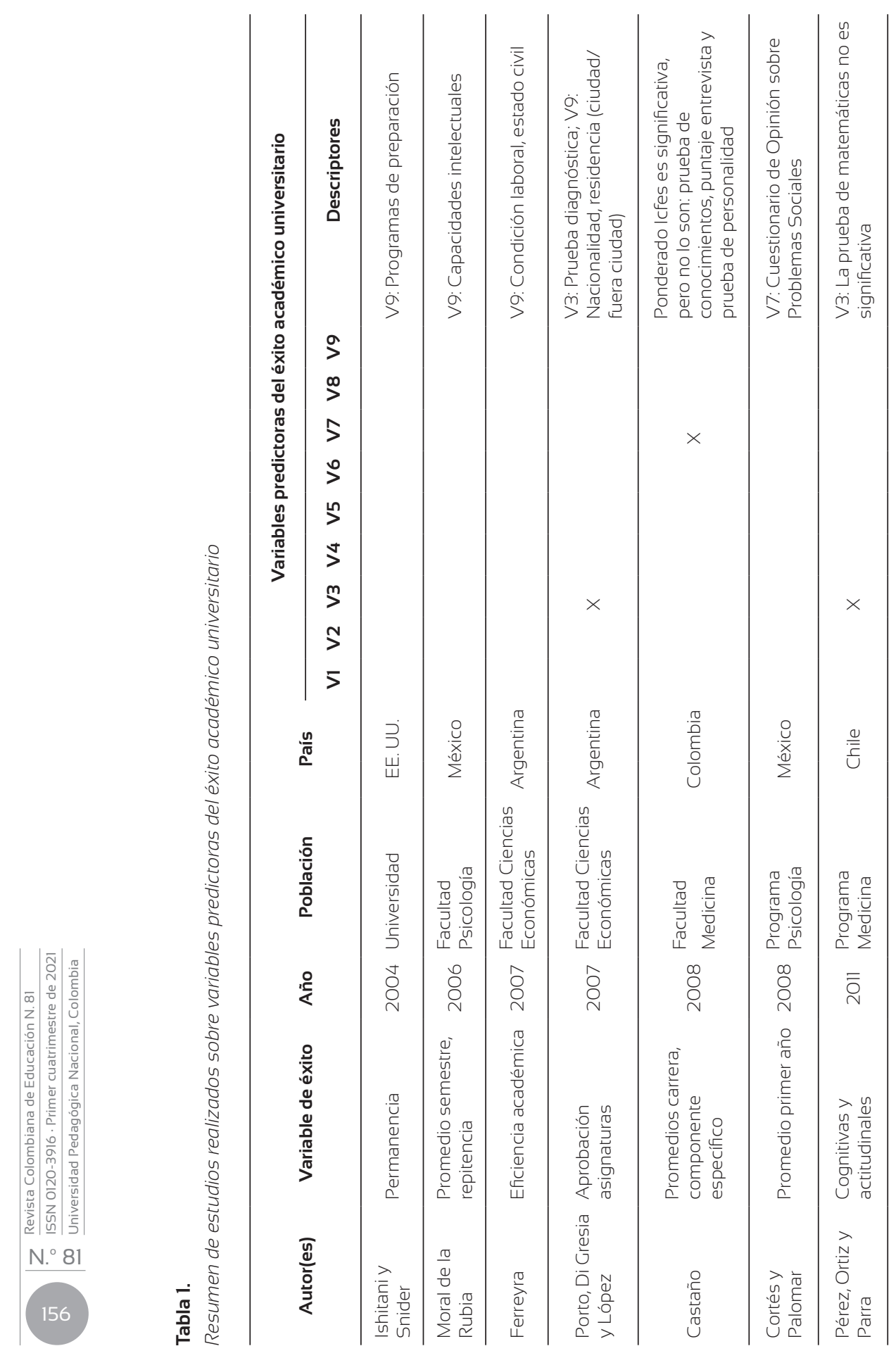



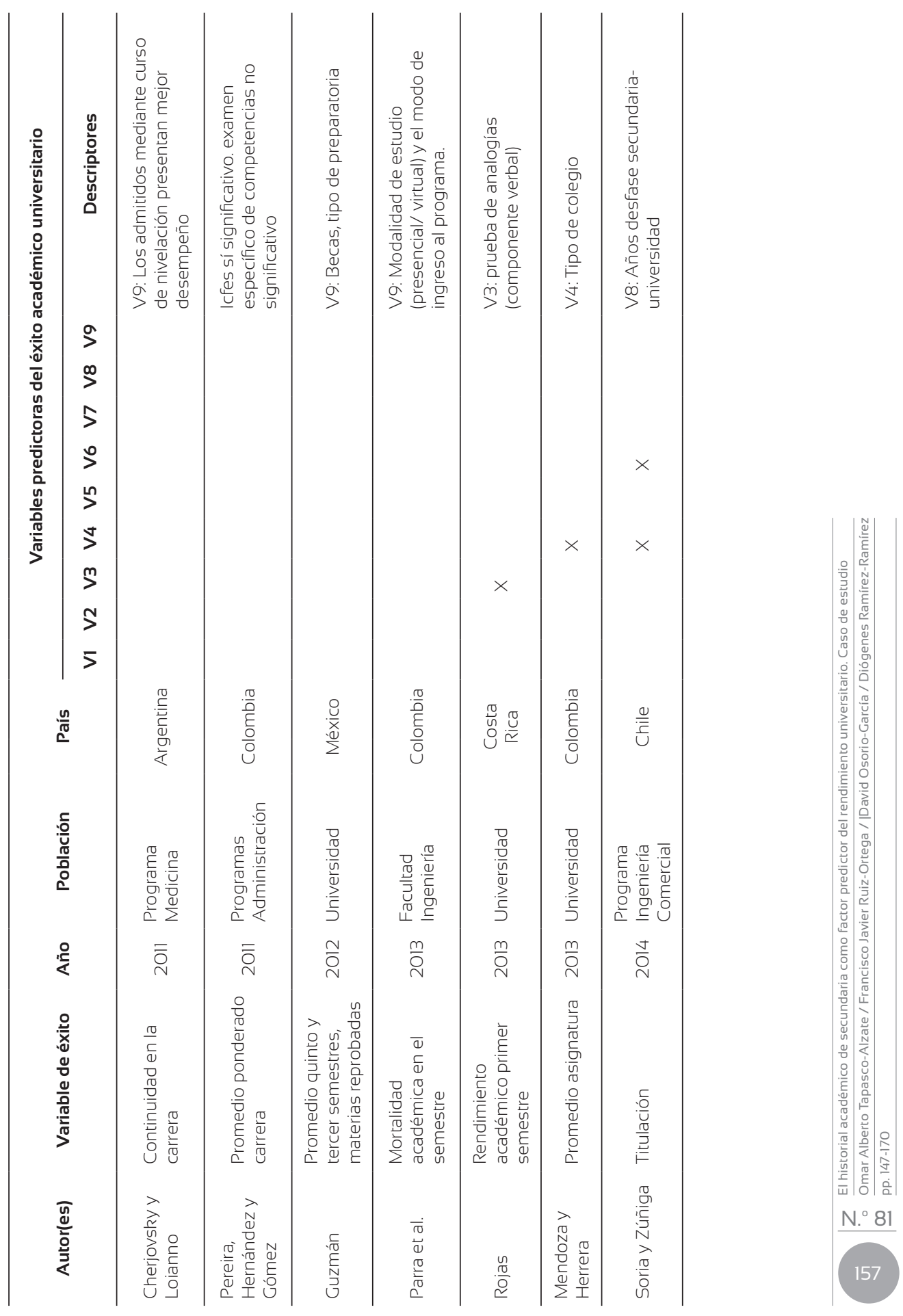


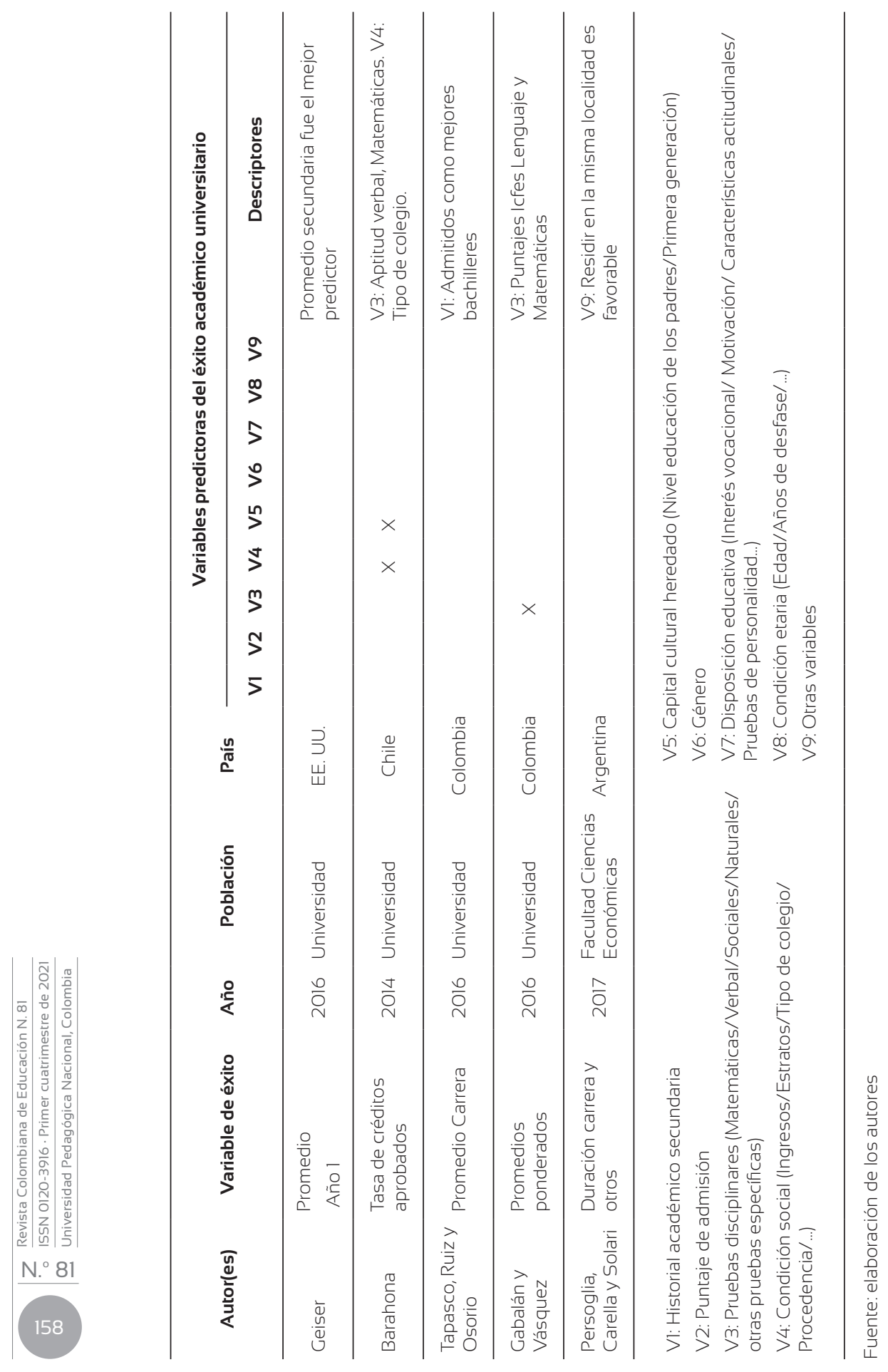


Es de anotar, en los trabajos revisados, una gran heterogeneidad, tanto en las variables de seguimiento como en las técnicas aplicadas. Así, algunas de ellas le hacen seguimiento al éxito académico desde la perspectiva del promedio en los primeros semestres de estudio o los promedios en ciertas áreas; otras, desde la permanencia en la institución y otras tantas desde la calificación promedio en la carrera. Además, el grupo de posibles variables predictoras no es el mismo en los distintos estudios, ya que algunas de las variables que son consideradas en unos estudios, no lo son en otros.

Cabe resaltar también cómo la variable que hace relación al historial académico de secundaria ha evidenciado un alto poder predictivo, esta resulta significativa en todos los estudios referenciados en donde se ha incluido como variable regresora, incluso marcando la mayor asociación con el desempeño universitario en muchos de ellos, tal como es señalado por Pérez, Ortiz y Parra (2011); Geiser (2016); Cerdeira et al. (2018); entre otros.

\section{Materiales y métodos}

La investigación realizada es de tipo transversal, con los datos recolectados para las cohortes 2011-1 y 2012-1, de los estudiantes de la Universidad de Caldas, Manizales (Colombia), de carácter correlacional, ya que apunta a evidenciar modelos predictivos del desempeño académico universitario. Se toma dicha población, pues para la fecha de estudio, este grupo de estudiantes ya tienen su situación académica definida, en términos de haberse graduado, o estar cerca de hacerlo o de haber desertado del programa al que fue admitido.

La metodología adoptada en la presente investigación contempla la utilización de modelos de regresión múltiple, haciendo uso del paquete estadístico $R$ (R Development Core Team, 2008), versión 3.4.3. La variable escogida para medir el éxito académico en la universidad fue el promedio acumulado de la carrera. Pero habida cuenta de que se detectaron diferencias significativas entre los promedios de los programas en la universidad se opta por obtener un valor estandarizado de dicha calificación, teniendo como referencia el promedio y la variabilidad dentro del programa, con el fin de compensar tales diferencias. En la tabla 2 se recopilan las variables empleadas en el estudio con sus respectivas categorías y rangos de respuesta, así como su escala de medición. 
Tabla 2.

Variables utilizadas en el estudio según la escala de medición y los niveles de respuesta.

\begin{tabular}{|c|c|c|}
\hline Variable & $\begin{array}{l}\text { Escala de } \\
\text { medición }\end{array}$ & Niveles \\
\hline $\begin{array}{l}\text { Puntaje de } \\
\text { admisión }\end{array}$ & Razón & $\begin{array}{l}\text { Puntaje calculado para cada programa, ponderado } \\
\text { según normativa de la universidad }\end{array}$ \\
\hline \multirow{2}{*}{$\begin{array}{l}\text { Opción de } \\
\text { admisión }\end{array}$} & \multirow{2}{*}{ Nominal } & Primera opción (1) \\
\hline & & Segunda opción (O) \\
\hline \multirow{2}{*}{ Sexo } & \multirow{2}{*}{ Nominal } & Masculino (O) \\
\hline & & Femenino (1) \\
\hline $\begin{array}{l}\text { Edad de ingreso } \\
\text { a la universidad }\end{array}$ & Razón & Edad al momento del ingreso \\
\hline Facultad & Nominal & Seis facultades \\
\hline $\begin{array}{l}\text { Calificación } \\
\text { promedio grado } 11\end{array}$ & Razón & $\begin{array}{l}\text { Dadas las distintas métricas de valoración en los } \\
\text { colegios, se decide escalar dichas calificaciones en un } \\
\text { rango de O a } 100\end{array}$ \\
\hline $\begin{array}{l}\text { Calificación } \\
\text { promedio del } \\
\text { programa }\end{array}$ & Razón & $\begin{array}{l}\text { Promedio acumulado en la carrera, estandarizado por } \\
\text { programas }\end{array}$ \\
\hline
\end{tabular}

Fuente: elaboración propia.

Dado que en las bases de datos de la universidad de donde se captura la información de los admitidos a ella, no se dispone del promedio que el estudiante obtiene en secundaria, se decide trabajar con una muestra de estudiantes de la universidad. Para ello se solicitó la información del promedio del estudiante en sus respectivos colegios. Dadas las limitaciones en la obtención de registros sistematizados de los datos de interés, se optó por seleccionar una muestra de conveniencia de instituciones de la ciudad. De los diez colegios consultados, cinco de ellos brindaron la información, dos de ellos privados y tres públicos, para recolectar en total la información del rendimiento académico de 266 estudiantes. Es de anotar que no se pudo obtener el historial académico completo, ya que solo proporcionaron información correspondiente al grado once de secundaria.

Para la medición de la incidencia de las variables predictoras, se hace uso del cálculo de la importancia relativa, que atañe a un concepto que ha sido de gran interés en años recientes, especialmente en trabajos de aplicación, y hace referencia a la cuantificación de la contribución de las variables regresoras en el modelo de regresión múltiple, la cual no se puede obtener de manera directa, dada la habitual multicolinealidad presentada en datos de este tipo (Grömping, 2006). 
Regularmente se acude al coeficiente de determinación como una medida de bondad de ajuste, ya que este se interpreta como el porcentaje de variabilidad en la variable respuesta que es explicada por las variables contempladas en el modelo. Particularmente, el cálculo de la importancia relativa cuando las variables explicativas están incorrelacionadas es directo, ya que corresponderá a la suma de los coeficientes de determinación de las regresiones individuales. Pero cuando existe multicolinealidad, el concepto de importancia relativa no está tan claramente definido. Una de las propuestas es acudir al promedio de las sumas de cuadrados secuenciales sobre todas las permutaciones de las variables explicativas.

Puntualmente, para el tratamiento de los datos obtenidos se acude al paquete relaimpo de $R$, el cual implementa la métrica ya mencionada, denominada $I m g$, y otras cuatro métricas adicionales que a continuación se describen. La métrica first usa la suma de cuadrados secuenciales cuando la variable de interés es ingresada de primera al modelo, mientras que la métrica last acude al mismo procedimiento, pero ingresando la variable al fina; por su parte, betasq hace una corrección con las desviaciones estándar al coeficiente estimado de la variable, y finalmente, la métrica pratt multiplica el coeficiente estandarizado con la correlación marginal (Grömping, 2006).

\section{Resultados y discusión}

A continuación, se resumen los estadísticos descriptivos de las variables incorporadas en el modelo, tanto de carácter cuantitativo (tabla 3), como de tipo categórico (tabla 4).

Tabla 3.

Estadísticos descriptivos variables cuantitativas

\begin{tabular}{lccccc}
\hline \multicolumn{1}{c}{ Variables } & N & Mínimo & Máximo & Media & D.E. \\
\hline Promedio grado 11 Colegio & 266 & 68,6 & 98,5 & 83,8 & 5,8 \\
\hline Edad de ingreso a la universidad & 266 & 16 & 22 & 17,9 & 0,9 \\
\hline Puntaje de admisión & 266 & 38,4 & 79,2 & 57,6 & 7,4 \\
\hline Promedio Carrera Estandarizado & 266 & $-3,8$ & 1,6 & $-0,03$ & 1,0 \\
\hline
\end{tabular}

Fuente: elaboración de los autores 
Tabla 4.

Frecuencias relativas de variables cualitativas

\begin{tabular}{lll}
\hline \multicolumn{1}{c}{ Variables } & \multicolumn{1}{c}{ Categorías } & Porcentajes \\
\hline \multirow{2}{*}{ Sexo } & Femenino & 42,0 \\
\cline { 2 - 3 } & Masculino & 58,0 \\
\hline \multirow{2}{*}{ Admitido } & Primera opción & 93,6 \\
\cline { 2 - 3 } & Segunda opción & 6,4 \\
\hline \multirow{3}{*}{ Facultad } & Agropecuarias & 8,0 \\
\cline { 2 - 3 } & Artes y Humanidades & 17,7 \\
\cline { 2 - 3 } & Ciencias Exactas y Naturales & 15,6 \\
\cline { 2 - 3 } & Ingeniería & 18,1 \\
\cline { 2 - 3 } & Jurídicas y Sociales & 25,0 \\
\cline { 2 - 3 } & Salud & 15,6 \\
\hline
\end{tabular}

Fuente: elaboración de los autores

Haciendo uso del análisis de regresión múltiple con las variables mencionadas, se estimó un modelo mediante el método paso a paso. De las seis posibles variables predictoras, solo dos mostraron significancia estadística: el promedio del colegio $(\mathrm{P}=3,01 \mathrm{E}-9)$ y el puntaje de admisión $(\mathrm{P}=0,0318)$. Al realizar las pruebas de verificación de los supuestos se encuentra que los datos analizados no cumplen los supuestos de normalidad y homocedasticidad, por lo que se procede a realizar una normalización de la variable respuesta restándole al valor dado el mínimo de los datos y dividiéndolo por el rango. Pero dado que la violación de los supuestos continúa presentándose, se decide realizar una transformación a los datos (Walpole, Myers, Myers y Ye, 2007). Con la función spreadLevelPlot del paquete car de $R$ se obtiene una transformación de potencia recomendada para la respuesta cuyo valor es 5,25. Su aplicación genera el cumplimiento de ambos supuestos, ya que el cálculo de la función gvlma del paquete gvlma de $R$ arroja aceptación del supuesto de normalidad $(P=0,1378)$ y del de homocedasticidad $(P=0,4746)$. $Y$ una prueba adicional para normalidad, test de Jarque-Bera, mediante el comando ajb.norm.test del paquete normtest de $R$, constata dicho cumplimiento $(\mathrm{P}=0,0765)$.

La tabla 5 recopila los resultados finales obtenidos de los parámetros estimados del modelo en donde se puede observar que las variables "promedio del colegio" ( $\mathrm{P}=5,37 \mathrm{E}-11)$ y "ponderado de admisión" ( $\mathrm{P}=0,0029)$ se mantienen como las variables significativas del modelo. 
Tabla 5.

Resumen de los coeficientes y sus significancias del modelo estimado

\begin{tabular}{lccccc}
\hline Variables & Coeficientes & $\begin{array}{c}\text { Error } \\
\text { estándar }\end{array}$ & Valor t & $\operatorname{Pr}(>|\mathrm{t}|)$ & $\begin{array}{c}\text { Códigos de } \\
\text { significancia }\end{array}$ \\
\hline Intercepto & $-1,6610$ & 0,2417 & $-6,87$ & $4,65 \mathrm{E}-11$ & $* * *$ \\
\hline $\begin{array}{l}\text { Ponderado } \\
\text { admisión }\end{array}$ & 0,0072 & 0,0039 & 3,00 & 0,0029 & $* *$ \\
\hline $\begin{array}{l}\text { Promedio } \\
\text { colegio }\end{array}$ & 0,0205 & 0,0030 & 6,85 & $5,37 \mathrm{E}-11$ & $* * *$ \\
\hline $\begin{array}{l}\text { R-cuadrado múltiple=0,2338, R cuadrado ajustado= 0,2279, Valor P= 9,163e-16 } \\
\text { (1) }\end{array}$ & \\
\hline
\end{tabular}

* Significativo al $95 \%$, ** Significativo al $99 \%$, *** Significativo al 99,9\%

Fuente: elaboración de los autores

Aunque el poder predictivo del modelo obtenido podría considerarse bajo desde la perspectiva del porcentaje de variabilidad explicada $(22,8 \%)$, muestra bastante concordancia con los obtenidos en otros trabajos similares, como el realizado por Rojas (2013) en donde obtienen un valor de 18,6\%, o el de Barahona (2014) con un $21 \%$, o como el obtenido por Geiser (2016), en donde se reporta un valor de 20,8 \% de variabilidad explicada, con el agregado de que en este estudio se toma el promedio consolidado de toda la carrera, mientras que en los anteriores se acude al rendimiento académico obtenido en los primeros semestres de estudios universitarios.

Dado que las variables predictoras evidencian estar correlacionadas entre sí, se acude al cálculo de la importancia relativa con el objetivo de evaluar el aporte que cada una de ellas tiene en el modelo predictivo obtenido. Y como se puede evidenciar en la tabla 6, los valores obtenidos de las métricas referenciadas anteriormente, presentan al promedio del colegio como la variable con mayor importancia relativa, presentando para todos los casos valores superiores al $68 \%$.

\section{Tabla 6.}

Importancia relativa de las variables explicativas en el modelo

\begin{tabular}{lccccc}
\hline & Img & last & first & betasq & pratt \\
\hline Ponderado admisión & 0,2612 & 0,1612 & 0,3157 & 0,1612 & 0,2294 \\
\hline Promedio colegio & 0,7387 & 0,8388 & 0,6843 & 0,8388 & 0,7706 \\
\hline
\end{tabular}

Fuente: elaboración de los autores 
El hecho de que las calificaciones de secundaria resultan ser un mejor predictor del desempeño en la universidad que las mismas pruebas estandarizadas, puede deberse a que el puntaje obtenido en una prueba estandarizada es el resultado de una única aplicación realizada en unas cuantas horas, lo que lo hace susceptible a factores que pueden distorsionar la puntuación real del desempeño de los evaluados, como la ansiedad que generan dichas pruebas (Olani, 2009), mientras que la nota promedio del bachillerato es la recopilación de múltiples evaluaciones realizadas a lo largo de un periodo de varios años (Geiser, 2016).

Al respecto, Duarte, Galaz y Rosales (2005) sugieren que un estudiante con buen rendimiento en el bachillerato cuenta con características, como hábitos y estrategias de estudio y actitudes hacia el aprendizaje, que le permiten desempeñarse académicamente mejor en sus estudios universitarios. En concordancia, Soria y Zúñiga (2014) reportan que las notas de enseñanza media efectivamente parecen indicar que estudiantes con buen desempeño académico antes de entrar a la universidad tienen mejores métodos de estudio, cierta disciplina y responsabilidad individual, características que muestran ser valoradas en el sistema universitario.

Si bien es cierto que los exámenes garantizan ciertos grados de abstracción y conocimiento en estándares mínimos, no son determinantes categóricos en posteriores desempeños, como bien se pudo constatar (Gabalán y Vásquez, 2016). Por ello, la práctica, tan común en Colombia, de acudir a los resultados del Icfes como medio de selección en los procesos de admisión a la universidad no debe ser el único referente, ni el más importante para el ingreso a la educación superior, dado su bajo poder predictivo del desempeño académico en la universidad (Pereira, Hernández y Gómez, 2011).

\section{Conclusiones y recomendaciones}

La presente investigación se propuso medir la incidencia de distintas variables en el desempeño académico del estudiante, teniendo como referencia de éxito, el promedio de notas obtenido en la carrera. Se encontró que solo dos variables evidencian una incidencia significativa, a saber: el promedio obtenido en el grado 11 y el puntaje de admisión a la universidad, el cual es el resultado de un promedio ponderado en las distintas áreas evaluadas en las pruebas de Estado. Al calcular la importancia relativa que cada una de estas variables tiene en el poder explicativo del modelo, arrojó que el desempeño académico de secundaria tenía un 73,9 \% de importancia; mientras que el puntaje de admisión el valor correspondiente era del $26,1 \%$. Tales resultados toman realce si 
se tiene en cuenta que una amplia literatura en esta temática señala al desempeño académico previo como una variable de alta incidencia en el desempeño futuro del estudiante universitario. Por lo que resultaría lógico proponer la incorporación del desempeño en la secundaria en los procesos de admisión a la universidad.

Adicional al incremento del poder predictivo, incluir el desempeño de bachillerato en los procesos de admisión enviaría una señal clara a los estudiantes de que trabajar duro y dominar las asignaturas en la educación secundaria puede ser una posibilidad directa para su ingreso a la universidad (Geiser, 2016). Además, minimizaría la necesidad de recurrir en gastos de preparación extracurriculares a pruebas estandarizadas en los que los estudiantes provenientes de familias con mayor poder adquisitivo obtienen una ventaja al lograr puntajes más altos en tales pruebas, lo que crea desigualdad en el acceso a la educación superior, sin que con ello se garantice un desempeño exitoso en la universidad (Prakhov, 2015). Aunque es importante anotar que lo anterior no implica que las pruebas estandarizadas sean irrelevantes, dado que los incentivos para esforzarse más a los que se enfrentan los estudiantes de educación media también están determinados por la existencia de aquellas (Cerdeira et ál., 2018).

Para hacer comparables los resultados de instituciones disímiles, se propone una normalización de las notas de secundaria, mediante el cálculo del percentil del rendimiento del estudiante en su institución, como un indicador de su desempeño relativo a su entorno, acompañados también por procesos de sistematización centralizados de la información, que permitan el acceso y la regulación de dicha información.

Finalmente, para proponer modelos de intervención en los sistemas de admisión en la educación superior en Colombia, se debe tener presente aquellas investigaciones que también señalan la acción en niveles de educación básica y media, para garantizar mejores resultados en las pruebas de Estado (principal mecanismo de acceso), y por otro lado, la asignación justa y equitativa de los recursos que permitan, una vez admitidos los estudiantes, garantizar su permanencia (Sánchez, Quirós, Reverón y Rodríguez, 2002) mediante los denominados cursos nivelatorios, el diagnóstico de competencias o saberes específicos que puedan ser el insumo para la intervención y cualificación de los procesos de aprendizaje de los estudiantes y por ende de los resultados en las pruebas de selectividad (Juarros, 2006). Otra posible acción surge por la implementación de políticas de acción afirmativa conducentes a equiparar las oportunidades para aquellos estudiantes en condiciones de desfavorecimiento. Aunque es importante anotar que las políticas de selección para el acceso a la educación superior han demostrado que no solo la acción afirmativa logra un mejor resultado sino su combinación con variables que miden el mérito académico (Cestau, Epple y Sieg, 2017). 


\section{Agradecimientos}

A las vicerrectorías Académica y de Investigaciones y Posgrados de la Universidad de Caldas, y a las entidades educativas Instituto Universitario; Instituto Tecnológico; Liceo Arquidiocesano Nuestra Señora Femenino; Fe y Alegría, y Liceo Isabel la Católica, de la ciudad de Manizales, por proveer el acceso a las calificaciones de los estudiantes que permitió el desarrollo de esta investigación. Al Programa de investigación Reconstrucción del tejido social en zonas de pos-conflicto en Colombia Código SIGP: 57579 con el proyecto de investigación "Hilando capacidades políticas para la transición en los territorios. Código SIGP: 57729". Financiado en el marco de la convocatoria Colombia Científica, Contrato No FP44842-213-2018.

\section{Referencias}

Aguirre, J. y Pabón, A. (2008). Sistemas especiales de admisión en las universidades: un desafío por la consolidación del derecho a la igualdad material en el acceso a la educación superior. Revista Estudios de Derecho, 66(146), 57- 83.

Bourdieu, P. y Passeron, J. (2009). Los herederos: los estudiantes y la cultura. Siglo xxı Editores.

Barahona, P. (2014). Factores determinantes del rendimiento académico de los estudiantes de la Universidad de Atacama. Estudios Pedagógicos, 40(1), 25-39.

Castaño, J. (2008). Correlación entre criterios de admisión y desempeño académico en estudiantes de la Facultad de Medicina de la Universidad de Manizales (Colombia). Archivos de Medicina, 8(2), 134-148.

Cestau, D., Epple, D. y Sieg, H. (2017). Admitting Students to Selective Education Programs: Merit, Profiling, and Affirmative Action. Journal of Political Economy, 125(3), 761-797. https://doi. org/10.1086/691702

Credé, M. y Kuncel, N. (2008). Study Habits, Skills, and Attitudes. The Third Pillar Supporting Collegiate Academic Performance. Perspectives on Psychological Science, 3(6), 425-453. https://doi.org/10.1111/j.17456924.2008.00089.x

Cerdeira, J.M., Nunes, L.C., Reis A.B. y Seabra C. (2018). Predictors of student success in Higher Education: Secondary school internal scores versus national exams. Higher Educ Q., 72, 304-313. https:// doi.org/10.1111/hequ.12158

Cherjovsky, M.R. y Loianno, M. (2011). Relación entre sistema de admisión y rendimiento académico. Revista Argentina de Educación Médica, 4(2), 55-62. 
Chica, S., Galvis, D. y Ramírez, A. (2010). Determinantes del rendimiento académico en Colombia. Revista Universidad EAFIT, 46(160), 48-72.

Cortés, A. y Palomar, J. (2008). El proceso de admisión como predictor del rendimiento académico en la educación superior. Universitas Psychologica, 7(1), 199-215.

Duarte, M., Galaz, J. y Rosales, J. (2005). Predictores de desempeño académico en una cohorte al finalizar el 2do. y 5to. Semestre en una Universidad Pública Estatal. Consultado el 15 diciembre de 2017 en http://www.comie.org.mx/congreso/memoria/v9/ponencias/atl6/ PRE1178912891.pdf

Esteban, M., Bernardo, A., Tuero, E., Cervero, A. y Casanova, J. (2017). Variables influyentes en progreso académico y permanencia en la universidad. European Journal of Education and Psychology, 10(2017), 75-81. http://dx.doi.org/10.1016/j.ejeps.2017.07.003

Ferreyra, M. (2007). Determinantes del desempeño universitario: efectos heterogéneos en un modelo censurado. [Tesis de Maestría en Economía]. La Plata, Argentina.

Gabalán, J. y Vásquez, F. (2016). Saber 11 y rendimiento universitario: un análisis del progreso en el plan de estudios. Ciencia, Docencia y Tecnología, (53), 135-161. Consultado el diciembre de 2017 en http://www.scielo.org. ar/scielo.php?script=sci_arttext\&pid=S1851-17162016000200007\&ln$\mathrm{g}=\mathrm{es} \& \ln \mathrm{ln}=\mathrm{es}$

Geiser, S. (2016). Medición y evaluación para los procesos de admisión de la educación superior: hallazgos desde California. Revista de Investigación Educacional Latinoamericana, 53(1), 1-18

Grömping, U. (2006). Relative Importance for Linear Regression in R: The Package relaimpo. Journal of Statistical Software, 17(1), 1-27.

González, A (2000). Precisiones conceptuales al principio de equidad. Pensamiento Educativo. 26, 15-29.

Guzmán, M. (2012). Modelos predictivos y explicativos del rendimiento académico universitario: caso de una institución privada en México. [Tesis doctoral]. Universidad Complutense de Madrid. Madrid, España.

Hoare, A. y Johnston, R. (2011). Widening participation through admissions policy- a British case study of school and university performance. Studies in Higher Education, 36(1), 21-41.

Ishitani, T. y Snider, K. (2004). Longitudinal Effects of College Preparation Programs on College Retention. Association for Institutional Research, 9(3), 1-10.

Juarros, M. (2006). ¿Educación superior como derecho o como privilegio? Las políticas de admisión a la universidad en el contexto de los países de la región. Andamios, 3(5), 69-90. http://www.scielo.org.mx/ scielo.php?script=sci_arttext\&pid=S1870-00632006000200005\&l$\mathrm{ng}=\mathrm{es} \& \operatorname{lng}=\mathrm{es}$ 
Mendoza, A. y Herrera, R. (2013). Propuesta para la predicción del rendimiento académico de los estudiantes de la Universidad del Atlántico, basado en la aplicación del Análisis Discriminante. World Engineering Education Forum, WEEF. Cartagena.

Moral de la Rubia, J. (2006) Predicción del rendimiento académico universitario. Perfiles Educativos, 28(113), 38-63.

Olani, A. (2009). Predicting First Year University Students' Academic Success. Electronic Journal of Research in Educational Psychology, 7(3), 1053-1072. http://www.redalyc.org/articulo.oa?id=293121984007

Parra, C., Mejía, L., Valencia, A., Restrepo, G., Usuga, O., y Mendoza, R. (2016). Rendimiento académico de los estudiantes de primer semestre de pregrado de la facultad de ingeniería de la universidad de Antioquia: cohorte 2012-2. Congresos CLABES. https://revistas.utp.ac.pa/index. php/clabes/article/view/883

Pereira, C.; Hernández, G. y Gómez, I. (2011). El valor predictivo de los exámenes de Estado frente al rendimiento académico universitario. Educación Educadores, 14(1), 51-65.

Pérez, C., Ortiz, L. y Parra, P. (2011). Prueba de selección universitaria, rendimiento en enseñanza media y variables cognitivo-actitudinales de alumnos de medicina. Revista Educación en Ciencias de la Salud, $8(2), 120-127$.

Persoglia, L., Carella, L. y Solari, E. (2017). Rendimiento académico y características socioeconómicas de graduados. Ciencia, Docencia y Tecnología, 28(54), 235-251.

Pinxten M., Van Soom C., Peeters C., De Laet T. y Langie G. (2017). At-risk at the gate: Prediction of study success of first-year science and engineering students in an open-admission university in Flanders. Any incremental validity of study strategies? European Journal of Psychology of Education, 33, 1-22.

Porto, A., Di Gresia, L. y López Armengol, M. (2007). Admisión a la universidad y rendimiento de los estudiantes. Estudio comparativo para estudiantes de Ciencias Económicas. Editorial de la Universidad de La Plata.

Prakhov, I.A. (2015). The Impact of Investments in Additional Preparation on Unified State Exam Results. Russian Education and Society, 57(5), 384-414.//dx.doi.org/10.1080/10609393.2015.1082415

Prakhov, I. y Yudkevich, M. (2017). University admission in Russia: Do the wealthier benefit from standardized exams? International Journal of Educational Development, 65, 98-105. https://doi.org/10.1016/j.ijedudev.2017.08.007

Pretz, J.E. y Kaufman, J.C. (2015). Do traditional admissions criteria reflect applicant creativity? The Journal of Creative Behavior, 51, 240-251. https://doi.org/10.1002/jocb.120 
R Development Core Team (2008). R: A language and environment for statistical computing. R Foundation for Statistical Computing. Vienna, Austria. http://www.R-project.org

Rivera, B. y Granobles, J. (2012). Análisis de políticas compensatorias para la equidad en el acceso a la educación superior: el caso de la Universidad de Caldas. El Pensamiento Universitario, (23), 81-91. Bogotá: Asociación Colombiana de Universidades.

Rojas, L. (2013). Validez predictiva de los componentes del promedio de admisión a la Universidad de Costa Rica utilizando el género y el tipo de colegio como variables control. Revista Electrónica Actualidades Investigativas en Educación, 13(1), 1-24.

Sánchez, A. y Otero, A. (2012). Educación y reproducción de la desigualdad en Colombia. Reportes del Emisor, Investigación e Información Económica, 154. Bogotá: Banco de la República.

Sánchez, F., Quirós, M., Reverón, C. y Rodríguez, A. (2002). Equidad social en el acceso y permanencia en la Universidad Pública. Determinantes y Factores Asociados. Centro de Estudios para el DesarroIlo Económico (CEDE), Universidad de los Andes, Bogotá. Soria, K. y Zúñiga, S. (2014). Aspectos determinantes del éxito académico de estudiantes universitarios. Formación Universitaria, 7(5), 41-50. http:// doi.org/10.4067/S0718-50062014000500006

Sulphey, M.M., Al Kahtani, N.S. y Abdul Malik Syed, A.M. (2018). Relationship between admission grades and academic achievement, Entrepreneurship and Sustainability Issues, 5(3), 648-658. http://doi. org/10.9770/jesi.2018.5.3(17)

Tapasco-Alzate, O., Ruiz-Ortega, F. y Osorio-García, D. (2016). Estudio del poder predictivo del puntaje de admisión sobre el desempeño académico universitario. Revista Latinoamericana de Estudios Educativos, 12(2), 148-165.

Taylor, R. (1999). Correlations between Differential Aptitude Test (DAT) scores and grade point average (GPA) for first year community college nursing students. http://proquest.umi.com/dissertations/ preview_ all/1395719

Ting, S.R. (2001). Predicting Academic Success of First-Year Engineering Student from Standardized Test Scores and Psychosocial Variables. Int. J. Engng Ed., 17(1), 75-80. https://www.ijee.ie/articles/Vol17-1/ ljee1168.pdf.

Vidigal, C.B.R. (2018). Racial and low-income quotas in Brazilian universities: impact on academic performance. Journal of Economic Studies, 45(1), 156-176. https://doi.org/10.1108/JES-10-2016-0200

Walpole, R., Myers, R., Myers, S. y Ye, K. (2007). Probabilidad y estadística para ingeniería y ciencias. Pearson Educación. 